\title{
Risk of Refractive Prediction Error After Cataract Surgery in Patients with Thyroid Eye Disease
}

\author{
Anne Strong Caldwell (iD' \\ Jennifer L Patnaik' \\ Melisa Ackerman' \\ Karen L Christopher (ID) \\ Anne M Lynch' \\ Jasleen K Singh ${ }^{2}$ \\ 'Department of Ophthalmology, \\ University of Colorado School of \\ Medicine, Aurora, CO, USA; \\ ${ }^{2}$ Department of Ophthalmology, \\ Children's Hospital of Colorado, Aurora, \\ CO, USA
}

Purpose: To investigate the risk of unexpected refractive prediction error after cataract surgery in patients with thyroid eye disease (TED) at the University of Colorado.

Patients and Methods: A retrospective observational study was performed using records of patients who underwent cataract surgery (2014 to 2018) who were included in a Cataract Surgery Outcomes database. Any patient with documentation of thyroid eye disease (TED) in the medical record was classified as TED. Post-operative refraction error greater than or equal to \pm 1.0 diopter from the target refraction was the main outcome of this study. Eyes with history of refractive surgery, ocular trauma, retinal detachment, non-Graves' disease thyroid conditions or Graves' disease without TED, and eyes without refractive error at follow-up were excluded.

Results: A total of 5716 eyes from 3692 patients who underwent cataract surgery were analyzed. Sixty-five eyes of thirty-nine patients (1.1\%) had TED. Former and/or current cigarette use was associated with having TED ( $\mathrm{p}=0.0504)$. Patients with TED had a statistically significant shorter axial length as compared to eyes without TED $(\mathrm{p}=0.0257)$. Three hundred and forty-nine eyes $(6.1 \%)$, including 9 eyes $(13.8 \%)$ in patients with TED, had refractive prediction error greater than \pm 1.0 diopter following surgery (univariate $\mathrm{OR}=2.5,95 \% \mathrm{CI}$ : 1.1-5.7, $\mathrm{p}=0.0274)$. After multivariate analysis controlling for race, tobacco use, combined surgery, and axial length, TED was associated with an increased risk of our primary outcome, refractive prediction error greater than \pm 1.0 diopter ( $\mathrm{OR}=2.4,95 \% \mathrm{CI}$ : $1.0-5.7, \mathrm{p}=0.0506)$.

Conclusion: Patients with TED are at increased risk for refractive prediction error following cataract surgery. Discussion with patients regarding their risk and possible need for glasses following surgery is important for setting realistic patient expectations.

Keywords: thyroid eye disease, cataract surgery, refractive surprise, graves disease

\section{Introduction}

Graves' disease affects $0.5 \%$ of the population and is the most common form of hyperthyroidism. ${ }^{1}$ Signs and symptoms of Graves' disease include weight loss, diarrhea, difficulty sleeping, tachycardia, and warm skin. Ocular manifestations of Graves' disease, termed thyroid eye disease (TED), can have a profound impact on the quality of life. ${ }^{2}$ Up to $20-35 \%$ of patients with Graves' disease will have concurrent TED at the time of diagnosis, ${ }^{3-5}$ and $80 \%$ of patients who develop TED will present within a 6-month window preceding or after diagnosis of Graves' disease. $^{5,6}$ In a recent meta-analysis, investigators estimated the prevalence of TED in patients with Graves' disease was $40 \%{ }^{7}$

The pathogenesis of TED is thought to be driven by an infiltrative autoimmune inflammatory process. Symptoms of TED range from grittiness, epiphora, and photophobia, to edema, lid retraction, proptosis, and diplopia. ${ }^{6,8}$ Severe TED can
Correspondence: Jasleen K Singh Department of Ophthalmology, Children's Hospital of Colorado, 13123 E 16th Ave, Mailstop B430, Aurora, CO, 80045, USA Tel +I 706-877-0869

Email Jasleen.singh@cuanschutz.edu 
manifest as compressive optic neuropathy (CON) or as corneal ulceration in the setting of exposure keratopathy. ${ }^{6,8}$ Recently, Chin et al reported a pooled prevalence of lid retraction and proptosis was 57\%, diplopia $36 \%$, and ocular hypertension $13 \%{ }^{7}$ Up to $40 \%$ of patients with Graves' disease and no clinically apparent ophthalmopathy will have subclinical extra-ocular muscle enlargement evidenced on imaging. ${ }^{9}$

Ocular co-morbidities including glaucoma, macular degeneration, diabetic retinopathy, and amblyopia have been documented as independent risk factors for unexpected refractive error after cataract surgery. ${ }^{10,11}$ While the general relationship between refractive error and Graves' Disease has been reported in the past, ${ }^{12}$ to our knowledge, no previous studies have shown a relationship between TED and unexpected refractive prediction error following cataract surgery. The purpose of our study was to determine if patients with a history of TED were at an elevated risk for unexpected refractive prediction errors after cataract surgery.

\section{Materials and Methods}

A retrospective cohort study was conducted using the Cataract Surgery Outcomes Database developed by the Department of Ophthalmology at the University of Colorado School of Medicine. ${ }^{13}$ The database has detailed information on clinical risk factors, intra-operative events, and cataract surgery outcomes at post-operative visits. All patients in the database underwent cataract surgery at the University of Colorado Sue Anschutz-Rodgers Eye Center. Patients who had bilateral surgery had both eyes included in the database. Data abstraction was performed retrospectively by Professional Research Assistants trained in cataract data abstraction. Quality control on this dataset included procedures for automatic range checks and an annual secondary review of at least $5 \%$ of the records by an ophthalmologist.

This study was approved by the Colorado Multiple Institutional Review Board and was conducted in compliance with the Declaration of Helsinki. Due to the retrospective nature of the study, a waiver of consent was approved. Patient data was kept confidential through the entire research process and anonymized after data extraction. Patients who underwent cataract surgery between January 2014 and December 2018 were included. The primary exposure for this cohort was a history of thyroid eye disease. For the purposes of this study, thyroid eye disease was classified by the patient's physician in the medical record. Each medical record was searched for terms "TED," "thyroid eye disease," "orbitopathy," "ophthalmopathy," and "graves." Patients with documentation of TED, thyroid associated orbitopathy, or thyroid associated ophthalmopathy were considered for TED in the cohort. Confirmation of TED was made with the presence of labs such as thyroid stimulating immunoglobulins or antithyroid antibodies documented in the record and/or documentation of the diagnosis from an ophthalmologist. All noted cases of TED were reviewed by ASC and by an ophthalmologist (JS) to confirm classification of the disease. Reviewers were masked to refractive outcomes.

The main outcome of the study was a large postoperative refraction prediction error, defined as spherical equivalent greater than or equal to \pm 1.0 diopter from the target refraction. Manifest refraction was measured at 6-meters using a phoropter. Refraction was collected between 21 and 120 days following cataract surgery with the best corrected visual acuity (BCVA) used for calculation of prediction error. Refraction prediction error was calculated by subtracting this measured refraction from the target refraction. The analysis concentrated on refractive prediction errors outside \pm 1.0 diopters due to the clinical significance of the threshold. ${ }^{15}$ Intraocular lens (IOL) selection was made using pre-operative measurements from the IOLMaster device (Carl Zeiss Meditec, Jena, Germany). The IOL was chosen based on the chosen refractive target after discussion with the patient, and target refraction was entered as the refraction predicted by the chosen IOL using the surgeon's formula selection (including Barrett Universal II, Hoffer Q, SRK/T, Holladay 1, and Holladay 2).

A large subset of patients was excluded if they had risk factors that could impact refraction outcomes. However, to maintain a substantial sample size of TED patients and ensure this patient group is properly representative, some risk factors that were similar between the TED group and controls were included. Patients with a history of corneal refractive surgery (LASIK, radial keratotomy, or photorefractive keratectomy), ocular trauma, history of retinal detachment, and those missing refractions in the window of 21-120 days following cataract surgery were excluded from the analysis. ${ }^{15}$ Furthermore, patients with history of other thyroid conditions, and those with Graves' disease but no TED were excluded to avoid possible confounding with misclassification due to under-diagnosed TED. There were no patients in the TED cohort who underwent orbital decompression within 3 months prior to their biometry 
measurements, and all refractions collected in the 21120 day timeframe occurred prior to any strabismus or orbital decompression surgery following their cataract surgery.

Demographic and clinical factors collected and analyzed for this study included gender, race/ethnicity, smoking status, whether it was a combined surgery, history of glaucoma, astigmatism (>2D), high hyperopia (preoperative spherical equivalent $>4 \mathrm{D}$ ), high myopia (preoperative spherical equivalent $<-6 \mathrm{D}$ or axial length $>26.5 \mathrm{~mm}$ ), age, BMI, axial length, TED and refractive error. Combined surgery was defined as phacoemulsification plus planned or unplanned vitrectomy, endoscopic cyclophotocoagulation, iStent, Baerveldt or Ahmed valve placement, dualblade goniotomy, or other additional procedures.

Basic frequencies were compared for eyes with a history of TED versus those without a history of TED, as well as eyes with the outcome of interest versus those without. Continuous variables were described with means and standard deviations (SD). Logistic regression models and odds ratios were used as the measure of association with $95 \%$ confidence intervals. P-values were calculated using generalized estimating equations to account for the intra-subject correlation of some patients having two eyes included. Potential risk factors that had p-values $<0.10$ in univariate associations with TED were included in the analysis of potential risk factors associated with the outcome. The multivariate model included TED as the primary explanatory variable and potential confounding factors.

\section{Results}

After exclusion criteria were applied, 5716 eyes from 3692 patients were included in the analysis. The clinical characteristics of the cohort are shown in Table 1. The mean age was 69.7 years, and the sex distribution was $53.2 \%$ female, $46.8 \%$ male. The cohort was predominantly nonHispanic white $(71.8 \%)$. A total of $54.8 \%$ of patients underwent cataract surgeries in both eyes. Thirty-nine patients and 65 eyes (1.1\%) had TED.

Patients with TED were significantly more likely to be female and African American (Table 2). A history of cigarette smoking or current smoking status were also associated with TED (OR $=2.0$ (95\% CI: 1.0-4.0, p = $0.0504)$ ). TED was not associated with glaucoma or preoperative astigmatism, pathological/high hyperopia, or high myopia. Axial length was shorter in eyes with history
Table I Clinical Characteristics of the Patients $(n=3692)$

\begin{tabular}{|l|c|c|}
\hline Characteristic & $\mathbf{n}$ & $\%$ \\
\hline Gender & 1727 & $46.8 \%$ \\
Male & 1965 & $53.2 \%$ \\
Female & & \\
\hline Race/Ethnicity & 2650 & $71.8 \%$ \\
Non-Hispanic White & 353 & $9.6 \%$ \\
African American & 325 & $8.8 \%$ \\
Hispanic & 180 & $4.9 \%$ \\
Asian & 110 & $2.9 \%$ \\
Other & 74 & $2.0 \%$ \\
Unknown & & \\
\hline Smoking Status & 1915 & $51.9 \%$ \\
Never & 1462 & $39.6 \%$ \\
Former & 310 & $8.4 \%$ \\
Current & 5 & $0.1 \%$ \\
Unknown & & \\
\hline Exposure of Interest & 2024 & $54.8 \%$ \\
Thyroid Eye Disease & & \\
\hline Repeated Subjects & & \\
Number of Patients with Two & 69.7 & $1.1 \%$ \\
Cataract Surgeries & 27.2 & \pm 5.8 \\
\hline Mean \pm Standard Deviation & & \\
Age & & \\
BMI (n=13I7) & & \\
\hline
\end{tabular}

Abbreviation: BMI, body mass index.

of TED $(23.7 \pm \mathrm{SD} 1.1 \mathrm{~mm})$ compared to those without a history of TED $(24.1 \pm$ SD $1.4 \mathrm{~mm}, \mathrm{p}=0.0257)$.

Mean post-operative BCVA was logMAR 0.078 (SD 0.15) for TED eyes and 0.076 (SD 0.19) for non-TED eyes (both Snellen equivalent around 20/24). The mean absolute prediction error was 0.43D (SD 0.37D) for the TED group and 0.40D (SD 0.47D) for the control group. Median absolute prediction error was also slightly larger for the TED group (0.34D) as compared to the control group (0.30D). Refraction prediction error greater than or equal to 1.0 diopters was present in $6.1 \%$ of the cohort $(\mathrm{n}=349)$ (Table 3). Within this group, 9 eyes were in patients with TED $(13.8 \%$, univariate $\mathrm{OR}=2.5(95 \%$ CI: $1.1-5.7, p=0.0274))$. Furthermore, combined surgery was significantly associated with refractive prediction error greater than or equal to 1.0 diopters $(10.4 \%$, univariate $\mathrm{OR}=2(95 \%$ CI: $1.5-2.6, \mathrm{p}<0.0001)$ ). In the univariate analysis, African Americans $(p=0.0827)$ and eyes that underwent combined surgery $(p<0.0001)$ experienced higher rates of refractive prediction error greater than $1.0 \mathrm{D}$. The average axial length in the group with refraction prediction error greater than 1.0 
Table 2 Relationship of Select Risk Factors with a History of Thyroid Eye Disease $(N=5716)$

\begin{tabular}{|c|c|c|c|c|c|c|c|c|}
\hline \multirow[b]{3}{*}{ Risk Factor } & \multicolumn{4}{|c|}{ History of TED } & \multirow[b]{3}{*}{ Total } & \multirow[b]{3}{*}{ OR } & \multirow[b]{3}{*}{$95 \% \mathrm{Cl}$} & \multirow[b]{3}{*}{$P$} \\
\hline & \multicolumn{2}{|c|}{ Yes } & \multicolumn{2}{|c|}{ No } & & & & \\
\hline & $\mathbf{n}$ & $\%$ & $\mathbf{n}$ & $\%$ & & & & \\
\hline \multicolumn{9}{|l|}{ Gender } \\
\hline Males & 17 & $0.7 \%$ & 2574 & $99.3 \%$ & 2591 & Ref & - & 0.0215 \\
\hline Females & 48 & $1.5 \%$ & 3077 & $98.5 \%$ & 3125 & 2.4 & $1.1-4.9$ & - \\
\hline \multicolumn{9}{|l|}{ Race/Ethnicity } \\
\hline Non-Hispanic White & 35 & $0.8 \%$ & 4089 & $99.2 \%$ & $4 \mid 24$ & Ref & - & - \\
\hline African American & 23 & $4.4 \%$ & 506 & $95.6 \%$ & 529 & 5.3 & $2.6-10.9$ & $<0.0001$ \\
\hline Hispanic & 6 & $1.2 \%$ & 493 & $98.8 \%$ & 499 & 1.4 & $0.4-4.8$ & 0.5715 \\
\hline Other/Unknown & 1 & $0.2 \%$ & 563 & $99.8 \%$ & 564 & 0.2 & $0.0-1.6$ & 0.1254 \\
\hline \multicolumn{9}{|l|}{ Smoking Status } \\
\hline Former/Current & 42 & $1.5 \%$ & 2699 & $98.5 \%$ & 2741 & 2.0 & $1.0-4.0$ & 0.0504 \\
\hline Never & 23 & $0.8 \%$ & 2947 & $99.2 \%$ & 2970 & Ref & - & - \\
\hline Unknown & 0 & $0.0 \%$ & 5 & $100 \%$ & 5 & NA & NA & NA \\
\hline \multicolumn{9}{|l|}{ Type of Surgery } \\
\hline Combined surgery & 14 & $1.9 \%$ & 709 & $98.1 \%$ & 723 & 1.9 & $0.9-4.0$ & 0.0877 \\
\hline Phacoemulsification only & 51 & $1.0 \%$ & 4940 & $99.0 \%$ & 4991 & Ref & - & - \\
\hline \multicolumn{9}{|l|}{ Glaucoma } \\
\hline Yes & 10 & $1.3 \%$ & 772 & $98.7 \%$ & 782 & 1.1 & $0.5-2.6$ & 0.7352 \\
\hline No & 55 & $1.1 \%$ & 4877 & $99.0 \%$ & 4932 & Ref & - & - \\
\hline \multicolumn{9}{|c|}{$\begin{array}{l}\text { Pre-Operative Astigmatism } \\
\geq 2 \mathrm{D} \mathrm{cyl}\end{array}$} \\
\hline Yes & 10 & $1.2 \%$ & 850 & $98.8 \%$ & 860 & 1.0 & $0.4-2.4$ & 0.9503 \\
\hline No & 55 & $1.1 \%$ & 4801 & $98.9 \%$ & 4856 & Ref & - & - \\
\hline \multirow{2}{*}{\multicolumn{9}{|c|}{$\begin{array}{l}\text { Pre-Operative High } \\
\text { Hyperopia } \geq 4 \mathrm{D}\end{array}$}} \\
\hline & & & & & & & & \\
\hline Yes & 2 & $3.7 \%$ & 52 & $96.3 \%$ & 54 & 3.4 & $0.5-25.7$ & 0.2322 \\
\hline No & 63 & $1.1 \%$ & 5599 & $98.9 \%$ & 5662 & Ref & - & \\
\hline \multicolumn{9}{|c|}{$\begin{array}{l}\text { Pre-Operative High Myopia } \\
\leq 6 \mathrm{D}\end{array}$} \\
\hline Yes & 9 & $0.9 \%$ & 1005 & $99.1 \%$ & 1014 & 0.7 & $0.3-1.7$ & 0.4858 \\
\hline \multirow[t]{3}{*}{ No } & 56 & $1.2 \%$ & 4646 & $98.8 \%$ & 4702 & Ref & - & - \\
\hline & \multicolumn{7}{|c|}{ History of TED } & \\
\hline & \multicolumn{4}{|c|}{ Yes } & \multicolumn{3}{|c|}{ No } & \\
\hline Age, mean $\pm S D$ & \multicolumn{4}{|c|}{$68.9 \pm 9.9$} & \multicolumn{3}{|c|}{$710.0 \pm 9.9$} & 0.4819 \\
\hline BMI, mean \pm SD & \multicolumn{4}{|c|}{$28.6 \pm 7.5$} & \multicolumn{3}{|c|}{$27.1 \pm 5.8$} & 0.3220 \\
\hline Axial Length, mean $\pm S D$ & \multicolumn{4}{|c|}{$23.7 \pm 1.1$} & \multicolumn{3}{|c|}{$24.1 \pm 1.4$} & 0.0257 \\
\hline
\end{tabular}

Abbreviations: BMI, body mass index; TED, thyroid eye disease.

D was slightly longer $(24.3 \pm$ SD $1.9 \mathrm{~mm}$ versus $24.1 \pm \mathrm{SD}$ $1.4 \mathrm{~mm}, \mathrm{p}=0.0087$ ).

The multivariate analysis was adjusted for race/ethnic group, smoking status, combined surgery, axial length, and TED. A total of 5526 records of the 5716 eyes are included in the multivariate analysis due to missing values for axial length. After adjusting for confounders, history of
TED was associated with 2.4 times increased adjusted odds of refractive prediction error greater than or equal to $1.0 \mathrm{D}(\mathrm{AOR}=2.4(95 \% \mathrm{CI}: 1.0-5.7, \mathrm{p}=0.0506))$.

\section{Discussion}

To the best of our knowledge there are no previous studies that have specifically examined the role of TED in 
Table 3 Association of Select Risk Factors with Refractive Errors Outside of \pm 1.0 Diopters

\begin{tabular}{|c|c|c|c|c|c|c|c|c|}
\hline \multirow[b]{3}{*}{ Risk Factor } & \multicolumn{4}{|c|}{ Refractive Error Outside \pm 1.0 Diopters } & \multirow[b]{3}{*}{ Total } & \multirow[b]{3}{*}{ OR } & \multirow[b]{3}{*}{$95 \% \mathrm{Cl}$} & \multirow[b]{3}{*}{$P$} \\
\hline & \multicolumn{2}{|c|}{ Yes } & \multicolumn{2}{|c|}{ No } & & & & \\
\hline & $\mathbf{n}$ & $\%$ & $\mathbf{n}$ & $\%$ & & & & \\
\hline Total population & 349 & $6.1 \%$ & 5367 & $93.9 \%$ & 5716 & NA & NA & NA \\
\hline \multicolumn{9}{|l|}{ Exposure of Interest } \\
\hline History of TED & 9 & $13.8 \%$ & 56 & $86.2 \%$ & 65 & 2.5 & I.I-5.7 & 0.0274 \\
\hline No History of TED & 340 & $6.0 \%$ & 5311 & $94.0 \%$ & 5651 & Ref & - & - \\
\hline \multicolumn{9}{|l|}{ Gender } \\
\hline Males & 156 & $6.0 \%$ & 2435 & $94.0 \%$ & 2591 & 1.0 & - & - \\
\hline Females & 193 & $6.2 \%$ & 2932 & $93.8 \%$ & 3125 & Ref & $0.8-1.3$ & 0.8202 \\
\hline \multicolumn{9}{|l|}{ Race/Ethnicity } \\
\hline Non-Hispanic White & 250 & $6.1 \%$ & 3874 & $93.9 \%$ & 4124 & Ref & - & - \\
\hline African American & 43 & $8.1 \%$ & 486 & $91.9 \%$ & 529 & 1.4 & $1.0-2.0$ & 0.0827 \\
\hline Hispanic & 24 & $4.8 \%$ & 475 & $95.2 \%$ & 499 & 0.8 & $0.5-1.2$ & 0.2998 \\
\hline Other/Unknown & 32 & $5.7 \%$ & 532 & $94.3 \%$ & 564 & 0.9 & $0.6-1.4$ & 0.7283 \\
\hline \multicolumn{9}{|l|}{ Smoking Status } \\
\hline Former/Current & 151 & $5.5 \%$ & 2590 & $94.5 \%$ & 2741 & Ref & - & - \\
\hline Never & 198 & $6.7 \%$ & 2772 & $93.3 \%$ & 2970 & 0.8 & $0.6-1.0$ & 0.0887 \\
\hline Unknown & 0 & $0 \%$ & 5 & $100 \%$ & 5 & NA & NA & NA \\
\hline \multicolumn{9}{|l|}{ Type of Surgery } \\
\hline Combined surgery & 75 & $10.4 \%$ & 648 & $89.6 \%$ & 723 & 2.0 & $1.5-2.6$ & $<0.0001$ \\
\hline \multirow[t]{3}{*}{ Phacoemulsification only } & 274 & $5.5 \%$ & 4717 & $94.5 \%$ & 4991 & Ref & - & \\
\hline & \multicolumn{7}{|c|}{ Refractive Error Outside \pm 1.0 Diopters } & \\
\hline & \multicolumn{4}{|c|}{ Yes } & \multicolumn{3}{|c|}{ No } & \\
\hline $\begin{array}{l}\text { Axial Length }(\mathbf{m m}) \text {, } \\
\text { mean } \pm S D\end{array}$ & \multicolumn{4}{|c|}{$24.3 \pm 1.9$} & \multicolumn{3}{|c|}{$24.1 \pm 1.4$} & 0.0087 \\
\hline
\end{tabular}

Abbreviation: TED, thyroid eye disease.

unexpected refractive prediction error after cataract surgery. Due to the complex nature of TED, it is important to understand any increased risk it might confer in surgical outcomes. A key finding of the study was that TED increases the risk of unexpected refractive prediction error after cataract surgery. In addition, other findings demonstrated that female gender and African-American race were significant risk factors for TED. Finally, in keeping with prior results, current or historical use of cigarettes was associated with TED in our cohort. ${ }^{16-18}$

Because of the disease pathology and the multiple surgeries that TED patients often receive, we hypothesized that patients with TED might be at increased risk for unexpected refractive prediction error after cataract surgery. Indeed, we found that patients with TED were more than twice as likely to have refraction prediction error outside of 1.0 D. Although the p-value of this association did not quite reach the threshold of $p<0.05$ for statistical significance, this is likely given the small sample size of eyes with TED in this study. The confidence interval indicates that we are $95 \%$ confident that the risk for clinically significant unexpected refractive prediction error greater than 1D ranges from 1.0 to or up to 5.7 times more likely in patients with TED than in those without. Such risk is almost certainly clinically significant when discussing risks and benefits of cataract surgery with a patient.

The pathophysiology of TED is complex, and many factors associated with the disease could play a part in the increased risk of refractive prediction error. Several investigators have described myopic, hyperopic, and cylinder axis changes in patients with TED independent from 
cataract surgery. Initially, the pathologic swelling and fibrosis of the muscles and soft tissues in the orbit is thought to alter the shape of the eye through compressive forces. ${ }^{19}$ In one study, adult Graves' disease patients were more myopic than the control group (mean spherical equivalent $-1.34 \pm 2.37 \mathrm{D}$ vs $0.31 \pm 0.77 \mathrm{D}, \mathrm{p}=0.001){ }^{12}$ They suggested the mechanism may be immune complex accumulation or enlarged extraocular muscle volume lateral to the eye, causing remodeling and thereby increasing the longitudinal axis of the eye. On the other hand, others have described a hyperopic shift in a patient with TED, hypothesized to occur due to posterior flattening of the globe in the setting of orbital compression. ${ }^{19}$ The differing reports speak to the complex and individual nature of each case and can likely be attributed to the unique disease course and anatomy of each eye and orbit.

Subsequent decompressive surgeries that adjust the position and shape of the eye in the orbit, and strabismus surgeries that change the tensile forces of the extraocular muscles may further affect the shape and therefore refractive status of the eye. Chandrasekaran et al described myopic shifts after decompressive surgery in each patient in a case series, and 2 of these patients had an increased axial length. ${ }^{19}$ These findings were corroborated by Kim et al who found that axial length (AL) significantly increased after orbital decompression surgery (change in $\mathrm{AL}=0.08 \mathrm{~mm} \mathrm{p}<0.001) .^{20}$

We hypothesize that the fluctuating volume status of the orbit in TED is a reason for the increased risk of unexpected refractive prediction error in patients with TED. Although TED is thought to classically manifest with an active inflammatory phase, followed by a plateau and an inactive phase, there have been several reports in the literature that might suggest TED flares associated with cataract surgery. $^{21-23}$ While the majority of patients in these reports received retrobulbar anesthesia, the bilateral nature of the presentations appears to be more consistent with a TED flare than an anesthetic-induced myotoxicity. It is possible that increased inflammation secondary to intraocular surgery and/or retrobulbar anesthesia triggered a new flare of TED. ${ }^{22}$ These reports demonstrate that even if cataract surgery is deferred until the fibrotic stage of TED, there may still be flares or fluctuations in the volume status of the orbit, thus causing change in refractive status between the biometry measurements and post-cataract surgery refraction. Furthermore, the unique orbital compressive forces that patients with TED face might alter the effective lens position in eyes with TED as compared to non-TED eyes with the same biometry measurements.

Given the high incidence of dry eye disease (DED) in patients with TED ${ }^{24}$ we hypothesize that DED may also play a part in increased risk for refractive prediction error following cataract surgery. The air-tear film interface of the cornea contributes greater than 40 diopters of refractive power to the eye. ${ }^{25}$ As such, variability of the ocular surface due to DED both during the time of keratometry but also on a day-to-day basis post-operatively and during refractions can cause fluctuations in measurements. In fact, Epitropoulos et al demonstrated that eyes with hyperosmolar tear film were more likely to have different intraocular lens strengths predicted on subsequent measurements than eyes with normal tear film osmolarity. ${ }^{26}$ In addition, DED can be further exacerbated during intraocular surgery resulting in increased dry eye symptoms and poor visual outcomes. $^{27-29}$ Pre-operative optimization of the tear film is important for optimal post-operative outcomes. ${ }^{29}$ Due to the high incidence of DED in TED patients it is especially important for this group.

In addition to the interplay of TED pathophysiology and its influence on refractive prediction error, we considered whether combined surgeries might impact our outcomes. Recently, Marta et al demonstrated that position of the Ahmed glaucoma valve can alter biometric predictability and expected final refraction. ${ }^{30}$ While combined surgeries can certainly influence refractive prediction error, in our cohort, the percent of those with TED who received combined surgery was similar to our control group (Table 2).

Limitations to our study include its retrospective nature and reliance on medical chart review. As with all retrospective studies where patient follow-up is important, we cannot rule out the possibility that patients with better outcomes were less likely to follow up, while those with worse outcomes were more likely to attend post-operative visits. Furthermore, our number of patients with TED was relatively small. Choice of IOL formula in our study also varied by surgeon preference; while it would be valuable to compare results between TED eyes and non-TED eyes using one of the newer-generation formulas for all eyes, doing so was outside of the scope of this study. The intent of this research was to evaluate whether large prediction error was more common in the TED group, and as formula choice variability was similar between the TED and non-TED group, one would expect a similar increase in prediction error regardless of formula choice. Duration of TED and severity as variables associated with risk of refractive prediction error were 
not considered due to a paucity of diagnosis dates in patient records. Further, we relied on physician's diagnoses of TED in the patient record, therefore misclassification and heterogeneity of our TED group may be present.

Based on our study results, we recommend counseling patients with a history of TED about the moderately increased risk of an unexpected refractive prediction error and potential unplanned need for glasses following cataract surgery, as well as future changes in refractive state with fluctuating disease status or decompression surgery. Including this information in the patient discussion prior to cataract surgery will aid clinicians in moderating patient expectations. Patients with active TED should be treated and managed by their orbital surgeon and endocrinologist prior to cataract surgery. In addition, as inadequately treated dry eye may have contributed to refractive prediction error in the TED patients, fully optimizing the patient's ocular surface prior to cataract surgery may improve outcomes.

\section{Acknowledgments}

The abstract of this paper was presented at the University of Colorado School of Medicine 35th Annual Research Forum as a poster presentation talk with interim findings.

\section{Funding}

Support from a grant to the Department of Ophthalmology from Research to Prevent Blindness, Inc., New York, NY, and the Colorado Clinical \& Translational Sciences Institute (CCSTI) with the Development and Informatics Service Center (DISC) from NIH/NCRR.

\section{Disclosure}

The authors have no conflicts of interests in this work.

\section{References}

1. Brent GA. Graves' disease. $N$ Engl J Med. 2008;358(24):2594-2605. doi:10.1056/NEJMcp0801880

2. Gerding MN, Terwee CB, Dekker FW, Koornneef L, Prummel MF, Wiersinga WM. Quality of life in patients with Graves' ophthalmopathy is markedly decreased: measurement by the medical outcomes study instrument. Thyroid. 1997;7(6):885-889. doi:10.1089/thy.1997.7.885

3. Tanda ML, Piantanida E, Liparulo L, et al. Prevalence and natural history of Graves' orbitopathy in a large series of patients with newly diagnosed graves' hyperthyroidism seen at a single center. $J$ Clin Endocrinol Metab. 2013;98(4):1443-1449.

4. Lim SL, Lim AK, Mumtaz M, Hussein E, Wan Bebakar WM, Khir AS. Prevalence, risk factors, and clinical features of thyroid-associated ophthalmopathy in multiethnic Malaysian patients with Graves' disease. Thyroid. 2008;18(12):1297-1301. doi:10.1089/thy.2008.0044
5. Bartley GB, Fatourechi V, Kadrmas EF, et al. The chronology of Graves' ophthalmopathy in an incidence cohort. Am J Ophthalmol. 1996;121(4):426-434. doi:10.1016/S0002-9394(14)70439-8

6. Douglas RS, McCoy AN, Gupta S. Thyroid Eye Disease. Springer, New York; 2014.

7. Chin $\mathrm{YH}, \mathrm{Ng} \mathrm{CH}$, Lee $\mathrm{MH}$, et al. Prevalence of thyroid eye disease in Graves' disease: a meta-analysis and systematic review. Clin Endocrinol. 2020;93(4):363-374. doi:10.1111/cen.14296

8. Bahn RS. Graves' ophthalmopathy. $N$ Engl J Med. 2010;362 (8):726-738. doi:10.1056/NEJMra0905750

9. Enzmann DR, Donaldson SS, Kriss JP. Appearance of Graves' disease on orbital computed tomography. J Comput Assist Tomogr. 1979;3(6):815-819. doi:10.1097/00004728-197903060-00019

10. Lundström M, Dickman M, Henry Y, et al. Risk factors for refractive error after cataract surgery: analysis of 282811 cataract extractions reported to the European Registry of quality outcomes for cataract and refractive surgery. J Cataract Refract Surg. 2018;44(4):447-452. doi:10.1016/j.jcrs.2018.01.031

11. Kugelberg M, Lundström M. Factors related to the degree of success in achieving target refraction in cataract surgery: Swedish National Cataract Register study. J Cataract Refract Surg. 2008;34 (11):1935-1939. doi:10.1016/j.jcrs.2008.06.036

12. Jankauskiene J, Jarusaitiene D. Assessment of visual acuity, refraction changes, and proptosis in different ages of patients with thyroid diseases. Int J Endocrinol. 2012;2012:643275. doi:10.1155/2012/643275

13. Miller DC, Christopher KL, Patnaik JL, et al. Posterior capsule rupture during cataract surgery in eyes receiving intravitreal anti-VEGF injections. Curr Eye Res. 2020;46(2):1-6.

14. Menconi F, Marcocci C, Marinò M. Diagnosis and classification of Graves' disease. Autoimmun Rev. 2014;13(4-5):398-402. doi:10.1016/j.autrev.2014.01.013

15. Geiger MD, Patnaik J, Miller DC, et al. Ocular comorbidities as risk factors for refractive surprise following cataract surgery. Invest Ophthalmol Vis Sci. 2019;60(9):2072.

16. Khong JJ, Finch S, De Silva C, et al. Risk factors for Graves' orbitopathy; the Australian Thyroid-Associated Orbitopathy Research (ATOR) Study. J Clin Endocrinol Metab. 2016;101 (7):2711-2720. doi:10.1210/jc.2015-4294

17. Prummel MF, Wiersinga WM. Smoking and risk of Graves' disease. JAMA. 1993;269(4):479-482. doi:10.1001/jama.1993.03500040045034

18. Vestergaard P. Smoking and thyroid disorders-a meta-analysis. Eur $J$ Endocrinol. 2002;146(2):153-161. doi:10.1530/eje.0.1460153

19. Chandrasekaran S, Petsoglou C, Billson FA, Selva D, Ghabrial R. Refractive change in thyroid eye disease (a neglected clinical sign). $\quad B r \quad J$ Ophthalmol. 2006;90(3):307-309. doi:10.1136/ bjo.2005.078295

20. Kim WS, Chun YS, Cho BY, Lee JK. Biometric and refractive changes after orbital decompression in Korean patients with thyroid-associated orbitopathy. Eye. 2016;30(3):400-405. doi:10.1038/eye.2015.242

21. Shadpour JM, Menghani RM, Douglas RS, Goldberg RA, Tsirbas A. Reactivation of thyroid-associated orbitopathy after cataract surgery. Jpn J Ophthalmol. 2009;53(1):44-46. doi:10.1007/s10384-008-0607-x

22. Wai DC, Ho SC, Seah LL, Fong KS, Khoo DH. Severe Graves' ophthalmopathy after retrobulbar anesthesia for cataract extraction in a patient with mild stable thyroid eye disease. Thyroid. 2003;13 (8):823-826. doi:10.1089/105072503768499725

23. Yi BP, Leng SL, Kwang LB, Rootman J. Development of thyroid-related orbitopathy following cataract surgery. Orbit. 2009;28(6):383-387. doi:10.3109/01676830903071232

24. Ismailova DS, Fedorov AA, Grusha YO. Ocular surface changes in thyroid eye disease. Orbit. 2013;32(2):87-90. doi:10.3109/ 01676830.2013 .764440

25. Navarro R. The optical design of the human eye: a critical review. J Optom. 2009;2(1):3-18. doi:10.3921/joptom.2009.3 
26. Epitropoulos AT, Matossian C, Berdy GJ, Malhotra RP, Potvin R. Effect of tear osmolarity on repeatability of keratometry for cataract surgery planning. J Cataract Refract Surg. 2015;41(8):1672-1677. doi:10.1016/j.jcrs.2015.01.016

27. Kim P, Plugfelder S, Slomovic AR. Top 5 pearls to consider when implanting advanced-technology IOLs in patients with ocular surface disease. Int Ophthalmol Clin. 2012;52(2):51-58. doi:10.1097/ IIO.0b013e31824b4504

28. Li XM, Hu L, Hu J, Wang W. Investigation of dry eye disease and analysis of the pathogenic factors in patients after cataract surgery. Cornea. 2007;26(9 Suppl 1):S16-20. doi:10.1097/ ICO.0b013e31812f67ca
29. Chuang J, Shih KC, Chan TC, Wan KH, Jhanji V, Tong L. Preoperative optimization of ocular surface disease before cataract surgery. J Cataract Refract Surg. 2017;43(12):1596-1607. doi:10.1016/j.jcrs.2017.10.033

30. Marta A, Coelho J, Vieira R, et al. Biometric Predictability in Combined Cataract Surgery and Ahmed Glaucoma Valve Implantation Depending on Tube Position. Clin Ophthalmol. 2021;15:2037-2045.

\section{Publish your work in this journal}

Clinical Ophthalmology is an international, peer-reviewed journal covering all subspecialties within ophthalmology. Key topics include: Optometry; Visual science; Pharmacology and drug therapy in eye diseases; Basic Sciences; Primary and Secondary eye care; Patient Safety and Quality of Care Improvements. This journal is indexed on PubMed
Central and CAS, and is the official journal of The Society of Clinical Ophthalmology (SCO). The manuscript management system is completely online and includes a very quick and fair peer-review system, which is all easy to use. Visit http://www.dovepress.com/ testimonials.php to read real quotes from published authors. 